\title{
Performance of a detonation driven shock tunnel
}

\author{
Jiwei $\mathrm{Li}^{1}$, Yingxin Tan ${ }^{2}$ \\ School of Chemical and Environmental Engineering, North University of China, Taiyuan, China, 030051 \\ Qiu Wang ${ }^{3}$, Wei Zhao ${ }^{4}$, Pan $\mathrm{Lu}^{5}$, \\ State Key Laboratory of High Temperature Gas Dynamics, Institute of Mechanics, Chinese Academy of Sciences, \\ Beijing, China, 100190
}

\begin{abstract}
Because of the high costs of flight tests, ground test facilities are a necessity in hypersonic flow research. Compared with other impulse facilities, shock tunnels show their advantages in relatively large-size models and low operational costs. To continuously improve the capacities of shock tunnels, a new detonation-driven shock tunnel was developed in the Laboratory of High Temperature Gas Dynamics (LHD), Institute of Mechanics, Chinese Academy of Sciences. In this paper, performance of the detonation-driven shock tube is investigated, with the help of numerical calculation. Some key issues, including the filling of the gases, pressure loss caused by diaphragm rupture, and the nearly tailored status are discussed in detail.
\end{abstract}

\section{Introduction}

Hypersonic technology is one of the most important research frontiers for aerospace program. Unlike what is seen by vehicles in subsonic or supersonic flightsubsonic or supersonic flights, hypersonic vehicles encounter an aero-thermodynamic environment characterized by strong shocks and high temperatures, that are accompanied by the vibrational excitation, dissociation and even the ionization of gas molecules. These complex phenomena make it difficult for an accurate prediction of the aerodynamic environment around a particular vehicle, and it is also an important issue in the area of gas dynamics research[1], which needs in-depth investigation. Although research in hypersonic technology has made great progress with the development of theories and numerical computation methods for decades, the ground test is still the main means of hypersonic research.

In order to meet the requirements as determined by ground experiments of hypersonic vehicles, advanced hypersonic test facilities have been under development for more than 50 years[2]. A air-heated, combustion-heated,

\footnotetext{
${ }^{1}$ Student, School of Chemical and Environmental Engineering, North University of China, No.3 XueYuan road, 1306532444@qq.com

2 Professor, School of Chemical and Environmental Engineering, North University of China, No.3 XueYuan Road, 13934240901@163.com

3 Assistant Professor, State Key Laboratory of High Temperature Gas Dynamics, No.15 Beisihuanxi Road, wangqiu@imech.ac.cn

${ }^{4}$ Professor, State Key Laboratory of High Temperature Gas Dynamics, No.15 Beisihuanxi Road, zw@imech.ac.cn

${ }^{5}$ Student, State Key Laboratory of High Temperature Gas Dynamics, No.15 Beisihuanxi Road, lupan@imec.ac.cn
} 
and arc-heated hypersonic wind tunnels have been developed, as have heated-light-gas-driven, free-piston-driven and detonation-driven shock tunnels. Taking account of real gas effects on hypersonic flows and high enthalpy requirements, the shock tunnel would be the most promising facility to provide hypersonic test flows. But, a shock tunnel must incorporate a high performance driver due to the huge energy requirement. Among the existing driving techniques, the detonation drivers are capable of producing high enthalpy and high pressure test flows simultaneously with easy operation and low capital investment. The detonation-driven method was first proposed to solve this problem by Bird in 1957[3]. A detonation-driven shock tube was first designed and installed by $\mathrm{Yu}$ [4] [5]. The TH2 that operated in a backward-running mode was developed in Aachen, Germany[6]. Later, a larger detonation-driven shock tunnel named HYPULSE was installed in GASL, USA[2]. These detonation-driven shock tunnels can be operated to provide either stable test flows with a long driving time at a relatively low enthalpy level or high-enthalpy flows with a short test duration. Recent rapid progress shows that detonation-driven hypersonic test facilities represent a promising method for implementing hypersonic vehicle ground tests.

But, no single ground test facility can fully simulate the many aspects of hypersonic flight. The quality of the free-stream flow, Mach number, Reynolds number at altitude, and gas chemistry cannot be controlled simultaneously in any single facility,if at all. Nearly every tunnel suffers from various problems, each having unique advantages and disadvantages. In order to promote the fundamental study of aero-thermo-dynamics and engineering applications in hypersonic flight, it is necessary to extend and supplement the capabilities of existing detonation driven shock tunnels. Nowadays, a new detonation-driven shock tunnel named JF-X was developed at LHD. As described in this paper, preliminary experiments, associated with numerical simulation, were carried out to investigate several key problems which impact the performance of the JF-X shock tunnel. Firstly, a critical nozzle was designed to control the rate of filling of gas and shock tube conditions that extended the test duration were analyzed. Then, the pressure loss caused by the diaphragm rupturing was also investigated.

\section{Facility}

For a detonation shock tunnel, two modes of operation are usually used. The mode in which the ignition position is located at the end of driver section is called the forward-running mode. This mode is, usually used to generate high enthalpy flow. The other mode, in which the ignition is performed near the diaphragm, is called the backwardrunning mode, and was the mode selected in the new tunnel because of, its stable performance after detonation. The detonation wave is initiated near the main diaphragm, and propagates upstream of the driver section. Meanwhile, the reactive high pressure and high temperature gas behind the detonation would rupture the main diaphragm, and generate a strong incident shock, which propagates through the driven tube, producing the interface (contact surface) between the driver and driven gases. When the primary shock strikes the end of the driven section, it is reflected, generating a reservoir region of almost stationary, high pressure and heated air [7]. Based on these techniques, several detonation-driven shock tunnels have been developed in the State Key Laboratory of High Temperature Gas Dynamics (LHD), Institute of Mechanics, Chinese Academy of Sciences (CAS), the high-enthalpy shock tunnel (JF10), the long-test-duration hypervelocity shock tunnel (JF12)[8] and the high-enthalpy expansion tunnel(JF16). These shock tunnels can provide hypersonic flows of higher total temperature than JF8A, which uses the high pressure air as the drive source. Among these are, the improved JF10 shock tunnel extends the effective test time to more than $6 \mathrm{~ms}$ and provides conditions to conduct testing when the total temperature is about $10000 \mathrm{~K}$. The JF12 shock tunnel is capable of reproducing flight conditions at altitude of 25 to $50 \mathrm{Km}$ and of that covering the Mach numbers from 5 to 9 with a test duration of more than $100 \mathrm{~ms}$, and it has the ability to test full-sized or nearly fullsized hypersonic vehicles. The JF 16 expansion tunnel can generate a test flow of over $8300 \mathrm{~m} / \mathrm{s}$ and a total enthalpy up to $40 \mathrm{MJ} / \mathrm{Kg}$, with test durations being around 50 to $100 \mathrm{~ms}$. To extend and supplement the capacities of existing shock tunnels, with the main parameters shown in Table 1, a new detonation driven shock tunnel, named JF-X, was developed at LHD. A photo of the shock tunnel is shown in Figure 1. It is, a reflected shock tunnel, with the driver section made with $42 \mathrm{CrMo}$ steel under the maximum allowable pressure of $50 \mathrm{MPa}$, and the driven section with SUS304 stainless steel under a pressure of $30 \mathrm{MPa}$. Other parameters can also be found in Table 1. Several pressure transducers were mounted to measure the velocity and pressure in the shock tube.

JF-X shock tunnel operates with backward-running mode which has been studied in LHD [9]. To explain the principle of the backward-running mode, the wave diagram of the detonation shock tube is shown in Figure 2. The detonation wave is initiated near the main diaphragm and propagates leftward to the dump section. A Taylor expansion wave follows the detonation wave. Meanwhile, the high-temperature and high-pressure detonated product gas that remains in the driving section acts as the driver source for the shock tube. After the main diaphragm rupture, an incident shock wave propagates almost simultaneously rightward in the shock tube followed by the interface. It is reflected at the shock tube end wall as shown in figure 2. If the reflected shock wave crosses over the interface 
without any reflected wave, that is the ideal experimental condition expected. In this paper, the simulations don't contain the dump section or the nozzle.

Table 1 Facility comparison

\begin{tabular}{ccccc}
\hline Facility & JF8A & JF10 & JF12 & JF-X \\
\hline \multirow{2}{*}{ Driver section } & $11 \mathrm{~m}$ in length & $10 \mathrm{~m}$ in length & $99 \mathrm{~m}$ in length & $6.5 \mathrm{~m}$ in length \\
& $150 \mathrm{~mm}$ in diameter & $150 \mathrm{~mm}$ in diameter & $400 \mathrm{~mm}$ in diameter & $126 \mathrm{~mm}$ in diameter \\
Shock tube & $21 \mathrm{~m}$ in length & $12.5 \mathrm{~m}$ in length & $89 \mathrm{~m}$ in length & $6.6 \mathrm{~m}$ in length \\
& $155 \mathrm{~mm}$ in diameter & $100 \mathrm{~mm}$ in diameter & $720 \mathrm{~mm}$ in diameter & $126 \mathrm{~mm}$ in diameter \\
Operation mode & High pressure Air & Forward detonation & Backward detonation & Backward detonation \\
& Contoured & Conical & Contoured & Contoured \\
Nozzle & $5 \mathrm{~m}$ in length & $2 \mathrm{~m}$ in length & $15 \mathrm{~m}$ in length & $2.3 \mathrm{~m}$ in length \\
& $0.8 \mathrm{~m}$ in exit diameter & $0.5 \mathrm{~m}$ in exit diameter & $2.5 \mathrm{~m}$ in exit diameter & $0.5 \mathrm{~m}$ in diameter \\
Temperature $\mathrm{T}_{0}$ & $\sim 1100 \mathrm{~K}$ & $\sim 10000 \mathrm{~K}$ & $\sim 3500 \mathrm{~K}$ & $\sim 7000 \mathrm{~K}$ \\
\hline
\end{tabular}

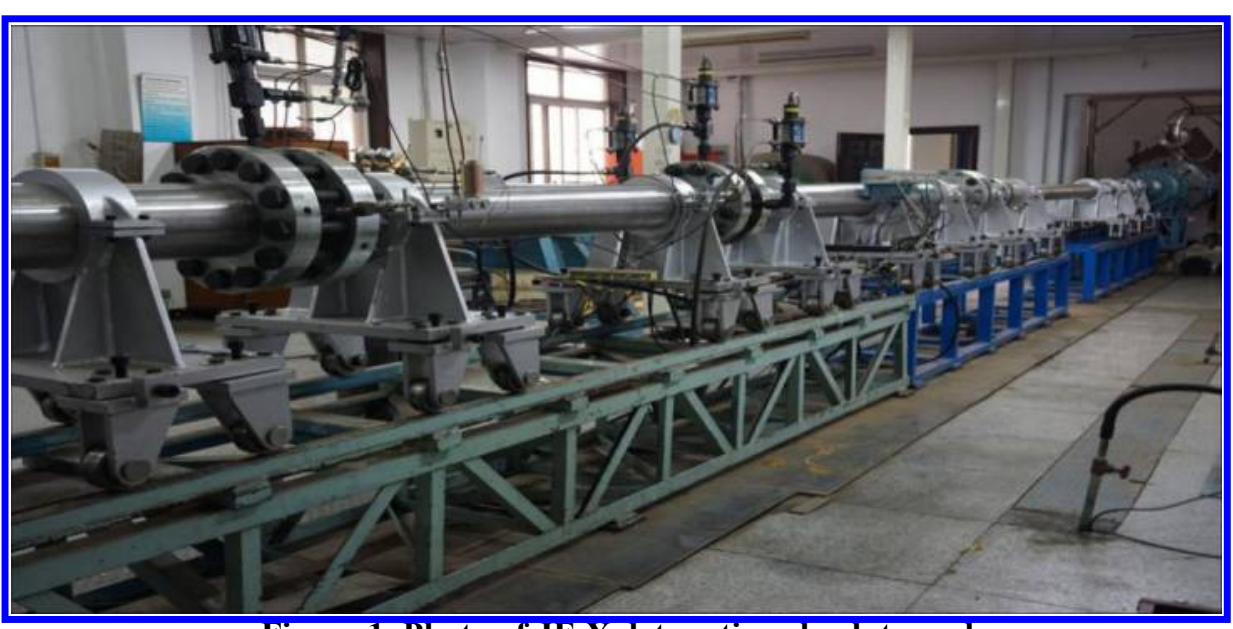

Figure 1. Photo of JF-X detonation shock tunnel

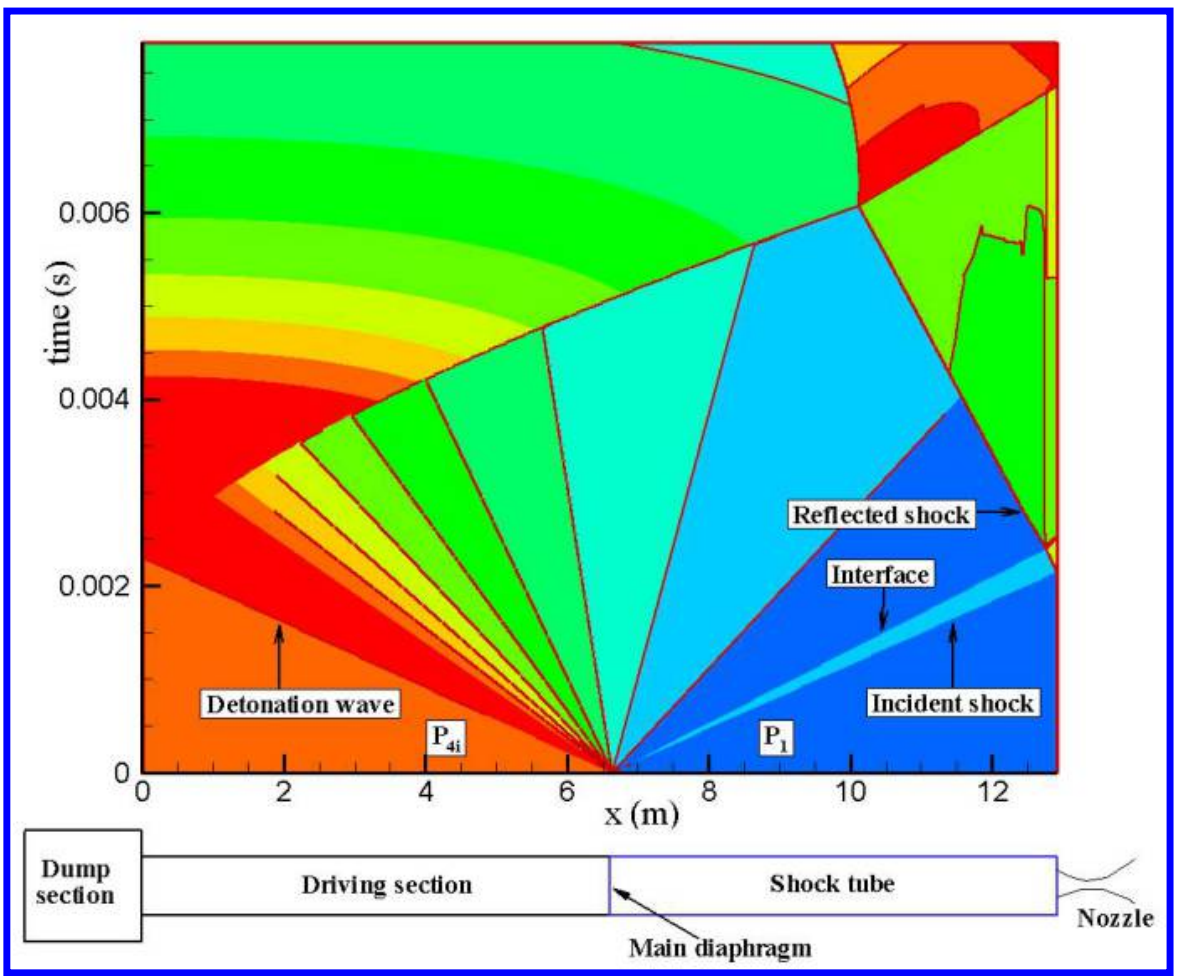

Figure 2. Schematic of the detonation driven shock tube and wave diagram 


\section{Numerical simulations}

To give a valuable complement to the experimental design and to better understand the wave process of the shock tube driven by detonation, numerical simulations were conducted for this paper. The shock tube involves detonation of the flammable mixtures in the detonation chamber and dissociation of the test gas (air in the paper) in the shock tube, which are rather complicated processes. Modeling all phenomena in detail is unrealistic. Also, the viscous term, heat conduction and rupture process of the diaphragm are neglected.

The numerical method is based on a one-dimensional chemical nonequilibrium flow model, with the equations, written in conservation form,

$$
A \frac{\partial U}{\partial t}+\frac{\partial A F(U)}{\partial x}-\frac{\partial A}{\partial x} H-S_{c}=0
$$

where the state vector $U=\left(\rho_{i}, \rho, \rho u, e, \rho \alpha, \rho \beta\right)^{T}$, the flux vector $F=\left(\rho_{i} u, \rho u, \rho u^{2}+p,(e+p) u, \rho \alpha u, \rho \beta u\right)^{T}$, the chemical reaction source term $S_{c}=\left(\dot{\omega}_{i}, 0,0,0, \dot{\omega}_{\alpha}, \dot{\omega}_{\beta}\right)$, and the wall pressure source term $H=(0,0, p, 0,0,0)^{T}$, where $\rho, u, e, p$, and $A$ are the density, velocity, total energy, and pressure of gas, and cross-sectional area, respectively. The subscript " $i$ " denotes the species $\left(\mathrm{O}_{2}, \mathrm{~N}_{2}, \mathrm{O}, \mathrm{N}\right), \alpha$ and $\beta$ are the process parameters of the chemical induction and the chemical transformation, respectively; $\omega_{\alpha}$ and $\omega_{\beta}$ are the rates of the chemical induction and the chemical transformation, respectively; and $\omega_{i}$ is the chemical source term for species $i$.

For the detonation process, a two-step chemical reaction model is used and $\rho_{i}$ is set to 0 in the computation. For the details of this model, please refer to reference [10]. Additional, the finite-rate chemistry developed by Park [11] is used for air in the shock tube without ionization, considering five components, $\mathrm{O}_{2}, \mathrm{~N}_{2}, \mathrm{O}, \mathrm{N}$, and NO . Based on these chemical reaction models and the dispersion controlled dissipation scheme proposed by Jiang [12], a code has been developed and successfully applied to the simulations of a detonation-driven shock tube [13].

\section{Some results and discussion}

\section{A. Filling of the gases}

The filling and mixing of the gases for detonation has an important effect on the stability and repeatability of the driving performance. In order to insure the ratio of oxy-hydrogen mixture, calibrations should be carried out before experiments. The filling system consists of a critical nozzle, a valve and a pressure regulator, as shown in Figure 3. It is based on the principle that when the nozzle reaches the speed of sound in the throat, the mass flow rate will not be affected by the pressure downstream. Then the mass flow rate is calculated by the following equation,

$$
m=\rho^{*} A^{*} a^{*}=P_{0} \sqrt{\frac{\gamma M}{R_{0} T_{0}}}\left(\frac{2}{\gamma+1}\right)^{\frac{\gamma+1}{2(\gamma-1)}} A^{*}
$$

where $\rho^{*}, a^{*}, P_{0}, \gamma, M, T_{0}$ represent density, sound speed, filling pressure, specific heat ratio, molecular weight and temperature of the filling gas; $A^{*}$ and $R_{0}$ are the area of the critical nozzle throat and the universal gas constant, respectively.

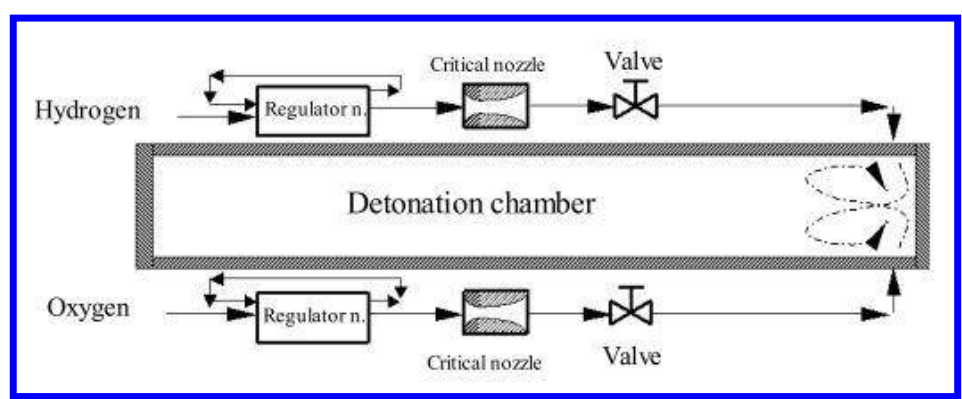

Figure 3. Illustration of filling and mixing oxy-hydrogen with critical nozzles 
It is assumed that the ratio of the oxy-hydrogen mixture is $n$, that is

$$
n=\frac{m_{1} / M_{1}}{m_{2} / M_{2}}
$$

where subscripts "1" and "2" represent hydrogen and oxygen respectively. Substituting equation (2) into equation (3), we get:

$$
n=\frac{\left(P_{0}\right)_{1}}{\left(P_{0}\right)_{2}} \times \sqrt{\frac{\left(T_{0}\right)_{2}}{\left(T_{0}\right)_{1}}} \times \frac{\left(A^{*}\right)_{1}}{\left(A^{*}\right)_{2}}
$$

Since the total temperature of the two gases is at room temperature and essentially unchanged during the filling process, the effects of temperature on $n$ are neglected here. Then,

$$
n=4 \frac{\left(P_{0}\right)_{1}}{\left(P_{0}\right)_{2}} \times \frac{\left(A^{*}\right)_{1}}{\left(A^{*}\right)_{2}}
$$

When $A^{*}$ is confirmed, it is easy to get a different value $n$ by changing $P_{0}$. Calibration of the hydrogen and oxygen systems is conducted with the results shown in Figure 4. Our target pressure in the driver section is $2.0 \mathrm{MPa}$ with $\mathrm{n}$ close to 2. The filling pressures are kept constant, which here are $5.5 \mathrm{MPa}$ for hydrogen and 4.1 MPa for oxygen, and much higher than the target pressure, in order to keep the velocity of sound at the critical nozzle throat. Monitoring the pressure changes in the driving tube, the filling rate and stability could be obtained, and are shown in Figure 4. During an exact experiment, the equivalence ratio of hydrogen and oxygen could be obtained during the filling process by monitoring the pressure changes of gas supply, as shown in Figure 5. The amount of hydrogen and oxygen in the driver section were increased linearly, and the ratio was almost constant during the entire filling process. The total experimental filling time was $397 \mathrm{~s}$, almost the same as that calculated through our calibration results, that was $395 \mathrm{~s}$. We can say that a needed initial gas condition in the driving tube could be exactly obtained by this filling system.

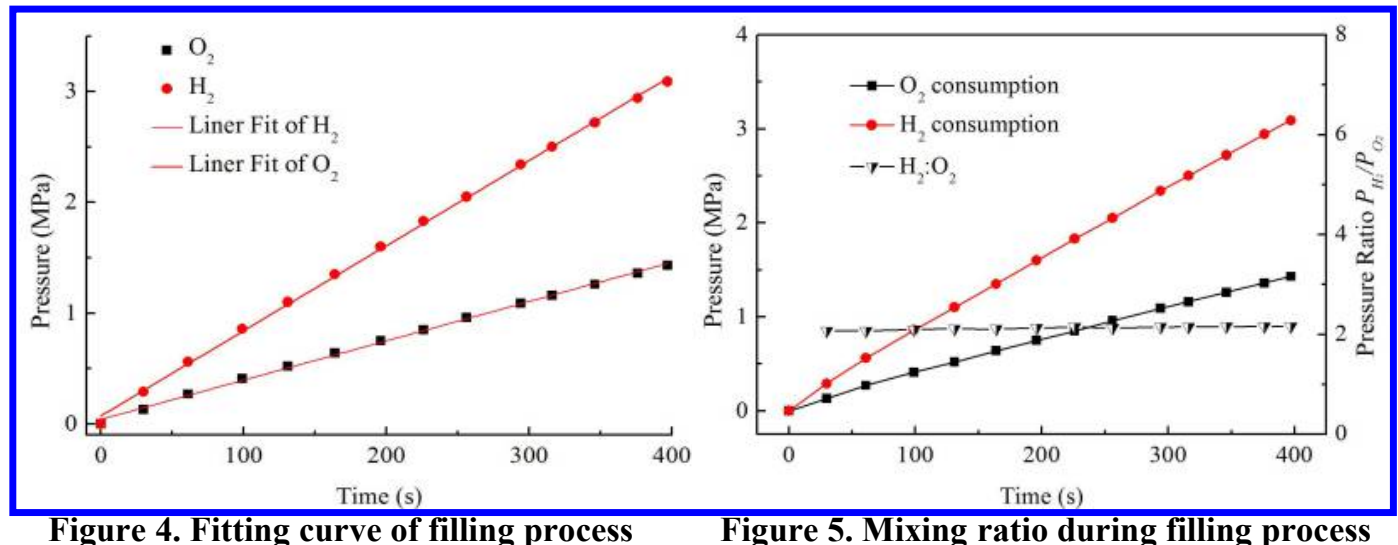

\section{B. Shock tube conditions}

According to the definitions of conventional shock tunnel terminology, subscripts " $4 \mathrm{i}$ " and " 1 " represent the initial condition of the driver section and the driven section respectively, subscript " 4 " represents the condition of detonation products. "Ms" represents the primary incident shock wave Mach-number in the shock tube and "a" is the local velocity of sound.

For a shock tunnel, effective test time is important. To lengthen the effective test time, it is expected that the reflected shock wave matches with the interface, which means that it crosses over the interface without any reflected wave. Then, a series of experiments were conducted to obtain a good performance of the facility, with the help of simulation. Test conditions are shown in Table 2. 
Table 2 Initial conditions

\begin{tabular}{ccc}
\hline & Driver Gas & Driven Gas \\
\hline Species & $\mathrm{H}_{2}, \mathrm{O}_{2}$ & Air \\
Ratio & 2.0 & - \\
Pressure & $\mathrm{P}_{4 \mathrm{i}}=2 \mathrm{MPa}$ & $\mathrm{P}_{1}=20,11.6,15 \mathrm{KPa}$ \\
\hline
\end{tabular}

Figures 6 and 7 show the typical stagnation pressure histories at the end wall of the shock tube, including both experimental and numerical results. The results were obtained under the conditions of initial pressure $P_{4 i}=2 \mathrm{MPa}$, the equivalence ratio is 2 and with different $P_{l}$. There was an obvious difference in the first $0.5 \mathrm{~ms}$ for the stagnation pressure, which then tended to the same value of almost $10 \mathrm{MPa}$ for the three cases. The plateau pressure continued for more than $6 \mathrm{~ms}$. The numerical results were slightly higher than the experimental results, which may be caused by the pressure loss while rupturing the metal diaphragm. Figure 8 shows the stagnation temperature histories of the numerical simulation. The results showed that the pressure of the driven section $P_{l}$ has a remarkable influence on the total temperature. It is About $6000 \mathrm{~K}$ and stays constant for more than $6 \mathrm{~s}$ while $P_{l}$ is $11.6 \mathrm{KPa}$.

The driving capacity was considered to be strongly influenced by the rupturing condition of the diaphragm placed between the driver section and shock tube [14]. Using the shock tube theory, the pressure ratio of $P_{4}$ to $P_{l}$ can be calculated by the following equation.

$$
\frac{P_{4}}{P_{1}}=\left[1+\frac{2 \gamma_{1}}{\gamma_{1}+1}\left(M_{s}^{2}-1\right)\right]\left[1-\frac{\gamma_{4}-1}{\gamma_{1}+1} \times \frac{a_{1}}{a_{4}}\left(M_{s}-\frac{1}{M_{s}}\right)\right]^{-\frac{2 \gamma_{4}}{\gamma_{4}-1}}
$$

With the detonation parameters obtained by a numerical calculation in section 2 , together with $M s$ and $P_{1}$, which could be accurately measured, it was easy to calculate $P_{4}$, which is the pressure effective for driving the gas in the shock tube. Then, the pressure loss by rupture of the diaphragm could be calculated with this $P_{4}$ and the detonation pressure by $\mathrm{CJ}$ theory. Experimental results show that the pressure loss was about $30 \%$ for selected diaphragm when the initial gas pressure was $2 \mathrm{MPa}$, with the mixing ratio of hydrogen and oxygen being 2 .
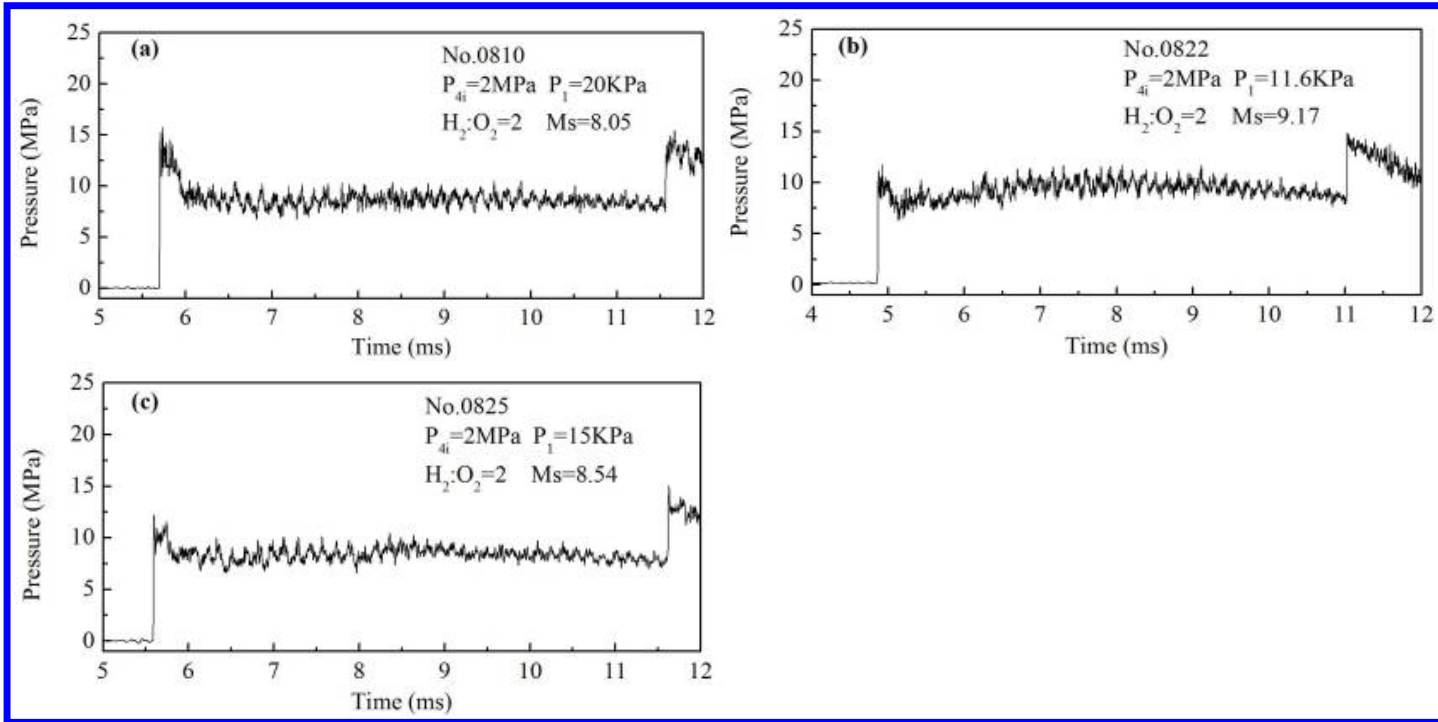

Figure 6. Stagnation pressure histories measured at the end wall of the driven section (a) $P_{1}=20 \mathrm{kPa}$, (b) $P_{1}=11.6 \mathrm{kPa}$, (c) $P_{1}=15 \mathrm{kPa}$ 


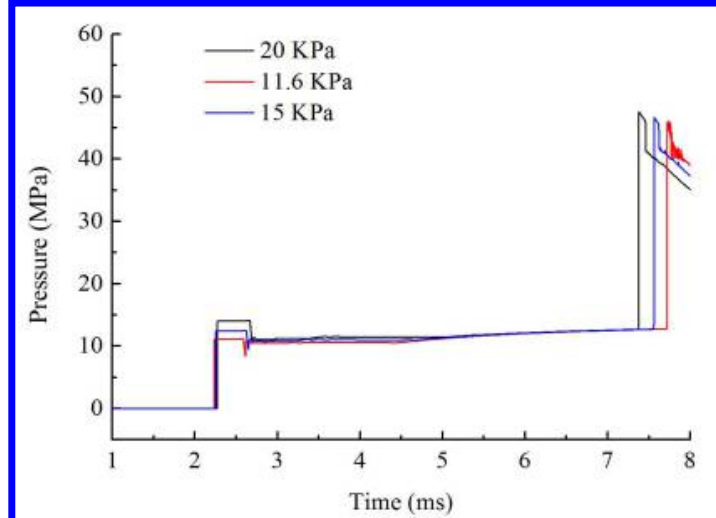

Figure 7. Simulated stagnation pressure histories under different values of $\boldsymbol{P}_{1}$

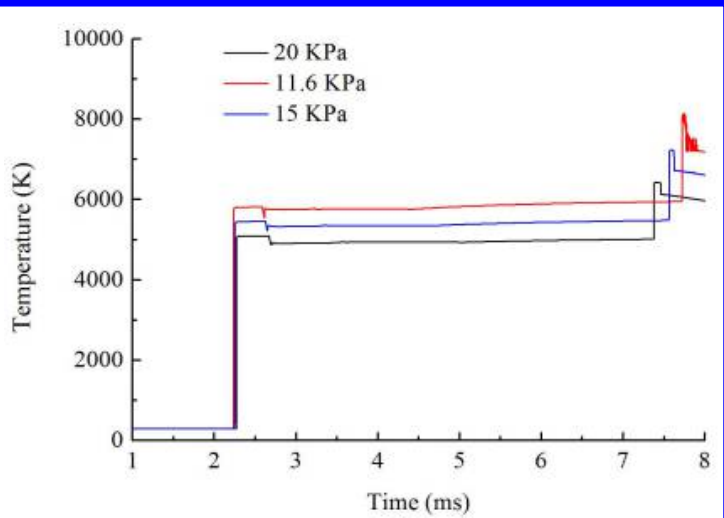

Figure 8. Simulated stagnation temperature histories under different values of $P_{1}$

\section{Pressure loss caused by diaphragm rupture}

Figure 9 shows the relationship between Mach number of the shock wave $M s$ and the different pressures of the driven section, $P_{l}$, under the condition for the initial pressure of the driver section, $P_{4 i}=2 \mathrm{Mpa}$, and with the equivalence ratio being 2 . The steel diaphragm was installed between the driver section and shock tube with an aggregate thickness of $3.4 \mathrm{~mm}$ and the effective thickness of $0.65 \mathrm{~mm}$. The Mach number of shock wave decreased as the pressure $P_{l}$ increased. But the Mach number as estimated by numerical simulation is markedly higher than the experimental results. Possible reasons for the lower Mach number include the ideal assumptions of the diaphragm opening instantaneously and the rupture period of the diaphragm in the simulation program. To take into account the influence of the diaphragm, define the non-dimensional pressure ratio, $P_{4} / P_{40}$, of value $P_{4}$ as calculated by using the experimental $M s$ to the value $P_{40}$ that is calculated by the simulation program. Figure 10 shows the relationship between $P_{4} / P_{40}$ and the pressure of the shock tube, $P_{1}$. The value of $P_{4} / P_{40}$ decreased lightly with increases of the initial pressure in the shock tube, but the actual driving pressure calculated by experimental $M s$ is almost 70 percent of the pressure of that calculated by $\mathrm{CJ}$ theory.

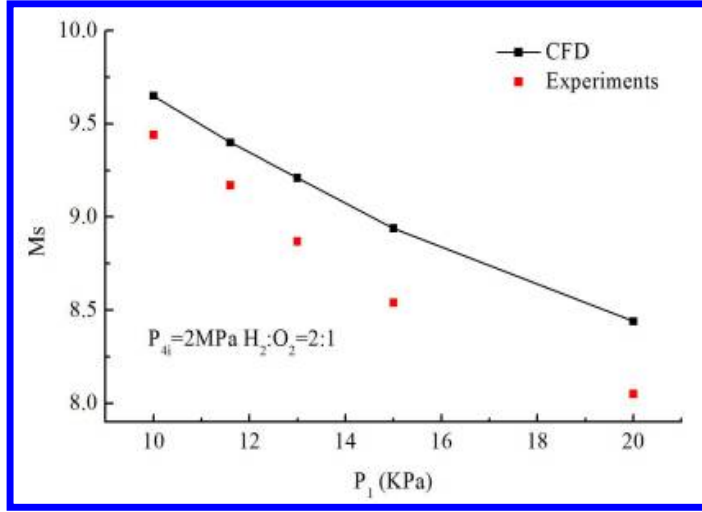

Figure 9. Relationship between Mach number of the shock wave $M s$ and the pressure of shock tube $P_{1}$

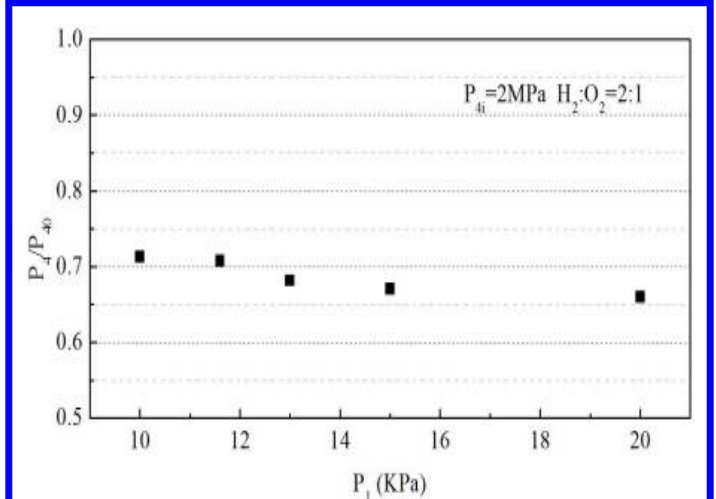

Figure 10. Relationship between pressure ratio $\boldsymbol{P}_{4} / \boldsymbol{P}_{40}$ and the pressure of the shock tube, $P_{1}$

\section{Conclusions}

A new detonation-driven shock tunnel was designed and installed at LHD. Operating in the backward-running mode, preliminary experiments were set up to clarify the performance of the filling of the gases and shock tube conditions. The influence of pressure loss caused by rupture of the diaphragm was also investigated. The results are summarized as follows.

By using the critical nozzle, the ratio of aeration could be controlled precisely and maintained at nearly a constant rate during the entire process. That is precious for insuring data accuracy and repeatability of experiments.

In the backward-running mode, the effective test time is more than $5 \mathrm{~ms}$, sufficient to provide the high enthalpy and pressure airflow required. That is, the JF-X detonation driven shock tunnel has enough capability to promote the desired aerodynamics experiments. 
The pressure loss caused by diaphragm rupture can not be neglected. For a specific diaphragm, a series of experiments should be launched to confirm the pressure loss under different initial conditions.

\section{Acknowledgments}

This work was supported by the National Natural Science Foundation of China (Grant Nos. 11402275, 11472280 and 11532014).

\section{References}

'Bertin J J, Cummings R M., "Fifty years of hypersonics: where we've been, where we're going," Progress in Aerospace Sciences, Vol 39, No. 6-7, 2003, pp. 511-536.

${ }^{2}$ Lu, F. K., Marren, D. E., Advanced Hypersonic Test Facility, Progress in Astronautics and Aeronautics, Reston, 2002 , p.198.

${ }^{3}$ Bird, G.A., A note on combustion driven tubes. Royal Aircraft Establishment, Vol 146, AGARD Report, May 1957

${ }^{4} Y$ u H R., Esser B., Lenartz M, Grönig, H., "Gaseous detonation driver for a shock tunnel," Shock Waves, Vol 2, No. 4, 1992 , pp. 245-254.

${ }^{5} \mathrm{Yu}$, H. R., Chen, H., Zhao, W., "Advances in detonation driving techniques for a shock tube/tunnel," Shock Waves, Vol 15, No. 6, 2006, pp. 399-405.

$\checkmark 6$ Vennemann, D., "Hypersonic test facilities available in Western Europe for aerodynamic/aerothermal and structure/material investigations," Philosophical Transactions of the Royal Society A Mathematical Physical \& Engineering Sciences, Vol 357, No. 357, 1999, pp. 2227-2248.

${ }^{7}$ Zhao, W., Jiang, Z. L., Saito, T., Lin, J. M., Yu, H. R., Takayama, K., "Performance of a detonation driven shock tunnel," Shock Waves, Vol 14, No. 1, 2005, pp. 53-59.

${ }^{8}$ Jiang, Z. L., Li, J. P., Zhao, W., Liu, Y. F., Yu, H. R., "Investigating into techniques for extending the test-duration of detonation-driven shock tunnels," Chinese Journal of Theoretical \& Applied Mechanics, Vol 44, No. 5, 2012, pp. 824-831.

${ }^{9} \mathrm{Yu}, \mathrm{H}$. R., Zhao, W., "The use of oxy-hydrogen detonation driver for generation of high enthalpy flow," 20th International Symposium on Rarefied Gasdynamics, Beijing, China, 1997, pp. 927-933

${ }^{10}$ Sichel M., Tonello, N. A., Oran, E. S., Jones, D. A., "A two-step kinetics model for numerical simulation of explosion and detonation in $\mathrm{H}_{2}-\mathrm{O}_{2}$ mixtures," Proceedings of the Royal Society A Mathematical Physical and Engineering Sciences, Vol 470, No. 2168, 2014, pp.49-82.

${ }^{11}$ C. Park., "Review of chemical-kinetic problems of future NASA missions, I: earth entries," Journal of Thermophysics and Heat Transfer, Vol 7, No. 3, 1993, pp. 385-398

12Jiang, Z. L., Takayama K., Chen, Y. S., "Dispersion conditions for non-oscillatory shock capturing schemes and its applications," Computational Fluid Dynamics Journal, Vol 4, No. 2, 1995, pp. 137-150.

${ }^{13} \mathrm{Li}$, J. P., Jiang, Z. L., Chen, H., "Numerical study on backward-forward double detonation driver for high-enthalpy shock tubes," Chinese Journal of Theoretical and Applied Mechanics, Vol 39, No. 3, 2007, pp. 343-349.

${ }^{14}$ Yamanaka, A., Ariga, Y., Obara, T., Cai, P., Ohyagi, S., "Study on Performance of Detonation-Driven Shock Tube," JSME International Journal, Vol 66, No. 45, 2000, pp.425-431. 